\title{
A note on isoperimetric inequalities of Gromov hyperbolic manifolds and graphs
}

\author{
Álvaro Martínez-Pérez ${ }^{1}$ (D) José $M$. Rodríguez $^{2}$ (D)
}

Received: 5 November 2020 / Accepted: 19 June 2021

(c) The Author(s) 2021

\begin{abstract}
We study in this paper the relationship of isoperimetric inequality and hyperbolicity for graphs and Riemannian manifolds. We obtain a characterization of graphs and Riemannian manifolds (with bounded local geometry) satisfying the (Cheeger) isoperimetric inequality, in terms of their Gromov boundary, improving similar results from a previous work. In particular, we prove that having a pole is a necessary condition to have isoperimetric inequality and, therefore, it can be removed as hypothesis.
\end{abstract}

Keywords Cheeger isoperimetric constant - Gromov hyperbolicity · Bounded local geometry $\cdot$ Pole

Mathematics Subject Classification 53C21 $\cdot 53 \mathrm{C} 23 \cdot 58 \mathrm{C} 40$

\section{Introduction}

It is well-known that isoperimetric inequalities are of interest in applied and pure mathematics $[22,48]$. The Cheeger isoperimetric inequality is related with many conformal invariants in

First author supported in part by a Grant from Ministerio de Ciencia, Innovación y Universidades (PGC2018-098321-B-I00), Spain. Second author supported in part by two Grants from Ministerio de Economía y Competitividad, Agencia Estatal de Investigación (AEI) and Fondo Europeo de Desarrollo Regional (FEDER) (MTM2016-78227-C2-1-P and MTM2017-90584-REDT), Spain. Also, the research of the second author was supported by the Madrid Government (Comunidad de Madrid-Spain) under the Multiannual Agreement with UC3M in the line of Excellence of University Professors (EPUC3M23), and in the context of the V PRICIT (Regional Programme of Research and Technological Innovation). We would like to thank to Paloma Martín for her support during this research.

$\triangle$ Álvaro Martínez-Pérez

alvaro.martinezperez@uclm.es

José M. Rodríguez

jomaro@math.uc3m.es

1 Departamento de Análisis Económico y Finanzas, Universidad de Castilla-La Mancha, Avda. Real Fábrica de Seda, s/n., 45600 Talavera de la Reina, Toledo, Spain

2 Departamento de Matemáticas, Universidad Carlos III de Madrid, Avenida de la Universidad 30, 28911 Leganés, Madrid, Spain 
Riemannian manifolds and graphs, namely the exponent of convergence, the bottom of the spectrum of the Laplace-Beltrami operator, Poincaré-Sobolev inequalities, and the Hausdorff dimensions of the sets of both escaping and bounded geodesics in negatively curved surfaces $[4,13],[18$, p. 228], [23,27-31,43,46], [52, p. 333]. Isoperimetric inequality is also closely related to Ancona's project on the space of positive harmonic functions of Gromov-hyperbolic graphs and manifolds [7-9]. In fact, in the study of the Laplace operator on a hyperbolic manifold or graph $X$, Ancona obtained in those three last papers several interesting results, under the hypothesis that the bottom of the spectrum of the Laplace spectrum $\lambda_{1}(X)$ is positive. If we denote by $h(X)$ the isoperimetric constant of $X$, the well-known Cheeger's inequality $\lambda_{1}(X) \geq \frac{1}{4} h(X)^{2}$ guarantees that $\lambda_{1}(X)>0$ when $h(X)>0$ (see [17] for a converse inequality). Therefore, the results in this paper are useful in order to apply these Ancona's results: We provide the minimal hypotheses in order to guarantee $h(X)>0$ (in fact, we have a characterization of this fact), then $\lambda_{1}(X)>0$ and we can apply Ancona's results to ensure the existence of solutions for elliptic partial differential equations in graphs and manifolds, among many other results (also, recall that in fact $h(X)>0$ and $\lambda_{1}(X)>0$ are equivalent conditions for a large kind of graphs and manifolds).

There is a natural connection between isoperimetric inequality and hyperbolicity. Recall that one of the definitions of Gromov hyperbolicity involves some kind of isoperimetric inequality (see [3,34]).

The Cayley graph of the group $\mathbb{Z}$ is an example of hyperbolic graph without isoperimetric inequality. Cao proved in [21] that hyperbolicity implies isoperimetric inequality (with an extra hypothesis on the Gromov boundary).

In [41] we studied the relationship of hyperbolicity and Cheeger isoperimetric inequality in the context of graphs and Riemannian manifolds with bounded local geometry.

A celebrated theorem of Kanai [37] gives that quasi-isometries preserve several interesting properties (including isoperimetric inequalities) between Riemannian manifolds and graphs with bounded local geometry. The isoperimetric inequality is also preserved with weaker hypotheses in the context of Riemann surfaces [20,33].

Mikhail Gromov started the theory of Gromov hyperbolic spaces in [34] in order to study finitely generated groups. The concept of Gromov hyperbolicity is very interesting since it grasps the essence of negatively curved Riemannian manifolds and discrete spaces like trees and the Cayley graphs of many finitely generated groups. Besides, Gromov hyperbolic spaces are an important class of metric spaces [14,16,19,32,54]. Gromov hyperbolicity has been thoroughly studied not only in Cayley graphs, but also in general graphs (see, e.g., $[5,6,11,12,15,39,40,47,49-51,55]$ and the references therein). Also, hyperbolicity has been applied in several areas such as the secure transmission of information and virus propagation on networks [35,36], real networks [1,2,24,38,45], or phylogenetics [25,26].

In [41] we characterized, in terms of their Gromov boundary, the uniform hyperbolic graphs and a large class of hyperbolic manifolds satisfying isoperimetric inequality (see, respectively, Theorems 2 and 4). In Theorems 2, 4 and [21, Theorem 1.1] it is used the hypothesis of the existence of a pole (in fact, although [21, Theorem 1.1] apparently uses a different hypothesis, actually, in hyperbolic spaces, it is equivalent to the existence of a pole). The hypothesis on Gromov hyperbolicity is natural (we need it in order to deal with the Gromov boundary). However, although the hypothesis on the pole is technically needed in the proofs, it does not look natural. The goal of this paper is to remove this hypothesis in the statements of Theorems 2 and 4. Thus, in Theorems 7 and 8 we prove that having a pole is not needed as an hypothesis but it is also a necessary condition of the characterization; this characterization appears in Theorems 5 and 6. 


\section{Background}

We include in this section the definitions and results that we will need in Sect. 3.

Definition 1 Given any Riemannian $n$-manifold $M$, the Cheeger isoperimetric constant of $M$ is defined as

$$
h(M)=\inf _{U} \frac{\operatorname{Vol}_{n-1}(\partial U)}{\operatorname{Vol}_{n}(U)},
$$

where $U$ ranges over all non-empty bounded open subsets of $M$, and $\operatorname{Vol}_{m}(S)$ denotes the $m$-dimensional Riemannian volume of $S$.

Along this paper we consider any graph $G=(V, E)=(V(G), E(G))$ endowed with its natural metric $d_{G}$ obtained by considering shortest paths (we always assume that every edge has length 1). Given any vertex $v \in V(G)$ and $m \in \mathbb{N}$ we denote, as usual, by $B_{G}(v, m)$, $\bar{B}_{G}(v, m)$ and $S_{G}(v, m)$ the open ball, the closed ball and the sphere in $G$ with center $v$ and radius $m$, respectively.

Definition 2 The combinatorial Cheeger isoperimetric constant of $G$ is defined to be

$$
h(G)=\inf _{U} \frac{|\partial U|}{|U|},
$$

where $U$ ranges over all non-empty finite subsets of vertices in $G, \partial U=\left\{v \in G \mid d_{G}(v, U)=\right.$ $1\}$ and $|U|$ denotes the cardinality of the set $U$.

A graph or Riemannian manifold $X$ satisfies the (Cheeger) isoperimetric inequality if $h(X)>0$, since this means that

$$
|U| \leq h(X)^{-1}|\partial U|,
$$

for every finite set of vertices $U$ if $X$ is a graph, and

$$
\operatorname{Vol}_{n}(U) \leq h(X)^{-1} \operatorname{Vol}_{n-1}(\partial U),
$$

for every bounded open set $U$ in the Riemannian $n$-manifold $X$.

We just consider graphs and manifolds $X$ which are connected (recall that if $X$ has connected components $\left\{X_{m}\right\}$, then $\left.h(X)=\inf _{m} h\left(X_{m}\right)\right)$.

Let $\left(X, d_{X}\right)$ and $\left(Y, d_{Y}\right)$ be two metric spaces, and $a, b$ constants satisfying $a \geq 1, b \geq 0$. A function $g: X \longrightarrow Y$ is an $(a, b)$-quasi-isometric embedding if

$$
\frac{1}{a} d_{X}\left(x_{1}, x_{2}\right)-b \leq d_{Y}\left(g\left(x_{1}\right), g\left(x_{2}\right)\right) \leq a d_{X}\left(x_{1}, x_{2}\right)+b,
$$

for every $x_{1}, x_{2} \in X$. We say that the function $g$ is $c$-full if for each $y$ in $Y$ there exists a point $x$ in $X$ with $d_{Y}(g(x), y) \leq c$.

A map $g: X \longrightarrow Y$ is a quasi-isometry, if there are constants $a \geq 1, b, c \geq 0$ such that $g$ is a $c$-full $(a, b)$-quasi-isometric embedding. Two metric spaces $X$ and $Y$ are said quasiisometric if there is a quasi-isometry $g: X \longrightarrow Y$. It is easy to see that to be quasi-isometric is an equivalence relation.

Definition 3 Given a graph $G$ and $v \in V(G)$, we denote by $N(v)$ the set of neighbors of $v$. The graph $G$ is $\mu$-uniform if each vertex $v$ of $V$ has at most $\mu$ neighbors, i.e., $\sup \{|N(v)|$ : $v \in V(G)\} \leq \mu$. We say that $G$ is uniform or that $G$ has bounded local geometry if it is $\mu$-uniform for some constant $\mu$. 
We say that a Riemannian $n$-manifold $M$ has bounded local geometry if there exist positive constants $r, a$, satisfying the following property: for each $p \in M$ there exists a diffeomorphism $F_{p}: B_{M}(p, r) \rightarrow \mathbb{R}^{n}$ such that

$$
\frac{1}{a} d\left(p_{1}, p_{2}\right) \leq\left\|F_{p}\left(p_{1}\right)-F_{p}\left(p_{2}\right)\right\| \leq a d\left(p_{1}, p_{2}\right)
$$

for every $p_{1}, p_{2} \in B_{M}(p, r)$.

If $M$ is a complete Riemannian manifold, the injectivity radius $\operatorname{inj}(x)$ of $x \in M$ is the largest radius for which the exponential map at $x$ is a diffeomorphism. If $M$ has non-positive sectional curvatures, then the injectivity radius can be defined, also, as the supremum of those $r>0$ such that $B_{M}(x, r)$ is simply connected or, equivalently, as half the infimum of the lengths of the (homotopically non-trivial) loops based at $x$. The injectivity radius $\operatorname{inj}(M)$ of $M$ is defined as

$$
\operatorname{inj}(M)=\inf _{x \in M} \operatorname{inj}(x) .
$$

One can check that $M$ has bounded local geometry if it has a lower bound on its Ricci curvature and positive injectivity radius [10].

Given a metric space $X$ and $x, y, w \in X$, we define the Gromov product of $x, y$ with respect to $w$ as

$$
(x \mid y)_{w}=\frac{1}{2}(d(x, w)+d(y, w)-d(x, y)) .
$$

Definition 4 Given $\delta \geq 0$ and a metric space $X$, we say that $X$ is $\delta$-(Gromov) hyperbolic if

$$
(x \mid y)_{w} \geq \min \left\{(x \mid z)_{w},(z \mid y)_{w}\right\}-\delta
$$

for every $x, y, z, w \in X$. We say that $X$ is hyperbolic if it is $\delta$-hyperbolic for some $\delta \geq 0$.

If $X$ is hyperbolic, we denote by $\delta(X)$ the sharp hyperbolicity constant of $X$ :

$$
\delta(X)=\inf \{\delta: X \text { is } \delta \text { - hyperbolic }\} .
$$

If $X$ is not hyperbolic, then we define $\delta(X)=\infty$.

Recall that a geodesic metric space $X$ is a metric space such that for every $x_{1}, x_{2} \in X$ there exists a geodesic joining them. A geodesic ray $\gamma$ emanating from $v \in X$ is a geodesic $\gamma:[0, \infty) \rightarrow X$ with $\gamma(0)=v$.

Definition 5 A geodesic metric space $X$ has a pole in a point $v$ if there exists $M>0$ such that each point of $X$ lies in an $M$-neighborhood of some geodesic ray emanating from $v$.

Definition 6 If $X$ is a geodesic metric space and $x_{1}, x_{2}, x_{3} \in X$, the union of three geodesics $\left[x_{1} x_{2}\right],\left[x_{2} x_{3}\right]$ and $\left[x_{3} x_{1}\right]$ is a geodesic triangle that will be denoted by $T=\left\{x_{1}, x_{2}, x_{3}\right\}$. We say that $x_{1}, x_{2}, x_{3}$ are the vertices of $T$. The geodesic triangle $T$ is $\delta$-thin if any side of $T$ is contained in the $\delta$-neighborhood of the union of the two other sides. We denote by $\delta_{t h}(T)$ the sharp thin constant of $T$, i.e., $\delta_{t h}(T):=\inf \{\delta \geq 0: T$ is $\delta-$ thin $\}$. We say that the space $X$ satisfies the Rips condition with constant $\delta$ (or is $\delta$-thin) if every geodesic triangle in $X$ is $\delta$-thin. We denote by $\delta_{t h}(X)$ the sharp thin constant of $X$, i.e., $\delta_{t h}(X):=\sup \left\{\delta_{t h}(T)\right.$ : $T$ is a geodesic triangle in $X\}$.

It is well-known that a geodesic metric space is hyperbolic if and only if it satisfies the Rips condition for some constant [3,32]. Although there are many different definitions of 
Gromov hyperbolicity (see, e.g., [3,32]), they are equivalent in the following sense: if $X$ is $\delta$-hyperbolic with respect to one definition, then it is $\delta^{\prime}$-hyperbolic with respect to another definition (for some $\delta^{\prime}$ related to $\delta$ ).

Since we consider any graph $G$ as a geodesic metric space, we need to identify any edge $u v \in E(G)$ with the interval $[0,1]$ in $\mathbb{R}$. Thus, the points in $G$ are the vertices and, also, the points in the interior of any edge of $G$. Therefore, any connected graph $G$ has a natural distance defined on its points (vertices and interior points), induced by taking shortest paths in $G$, and we can see $G$ as a metric graph.

Definition 7 Given a metric space $(X, d)$ and a constant $A>1$, we say that $(X, d)$ is $A$ uniformly perfect if there exists some $\varepsilon_{0}>0$ such that for every $0<\varepsilon \leq \varepsilon_{0}$ and every $x \in X$ there exist a point $y \in X$ such that $\varepsilon / A<d(x, y) \leq \varepsilon$. We say that $(X, d)$ is uniformly perfect if there exists some $A$ such that $(X, d)$ is $A$-uniformly perfect.

Let us recall the concept of boundary of a hyperbolic space. For further information we refer the reader to $[14,19,32,34]$.

Let $X$ be a hyperbolic space and fix $w \in X$.

A sequence of points $\left\{x_{i}\right\} \subset X$ converges to infinity if

$$
\lim _{i, j \rightarrow \infty}\left(x_{i} \mid x_{j}\right)_{w}=\infty
$$

One can check that this property is independent from the choice of $w$. Two sequences $\left\{x_{i}\right\},\left\{x_{i}^{\prime}\right\}$ that converge to infinity are equivalent if

$$
\lim _{i \rightarrow \infty}\left(x_{i} \mid x_{i}^{\prime}\right)_{w}=\infty
$$

One can check that this defines an equivalence relation for sequences in $X$ converging to infinity. The sequential boundary at infinity $\partial_{\infty} X$ of $X$ is the set of equivalence classes of sequences converging to infinity.

For every $\xi, \xi^{\prime} \in \partial_{\infty} X$, its Gromov product with respect to $w \in X$ is defined as

$$
\left(\xi \mid \xi^{\prime}\right)_{w}=\inf \liminf _{i \rightarrow \infty}\left(x_{i} \mid x_{i}^{\prime}\right)_{w},
$$

where the infimum is taken over all sequences $\left\{x_{i}\right\} \in \xi,\left\{x_{i}^{\prime}\right\} \in \xi^{\prime}$.

A metric $d$ on $\partial_{\infty} X$ is visual if there exist $w \in X, A>1$ and positive constants $k_{1}, k_{2}$, such that

$$
k_{1} A^{-\left(\xi \mid \xi^{\prime}\right)_{w}} \leq d\left(\xi, \xi^{\prime}\right) \leq k_{2} A^{-\left(\xi \mid \xi^{\prime}\right)_{w}}
$$

for all $\xi, \xi^{\prime} \in \partial_{\infty} X$. In this case, we say that $d$ is a visual metric with respect to $w$ and $A$.

Theorem 1 [19, Theorem 2.2.7] If $X$ is a hyperbolic space, then for every $w \in X$, there exists $A_{0}>1$ such that for each $A \in\left(1, A_{0}\right]$ there is a metric $d_{A}$ on $\partial_{\infty} X$, which is visual with respect to $w$ and $A$.

Note that $\partial_{\infty} X$ is a bounded and complete metric space for any visual metric.

Theorem 2 [41, Theorem 4.15] If $G$ is a hyperbolic uniform infinite graph with a pole, then $h(G)>0$ if and only if $\partial_{\infty} G$ is uniformly perfect for some visual metric.

Let $(X, d)$ be a metric space, $Y \subset X$ and $t>0$. The subset $Y$ is $t$-separated in $X$ if $d\left(y_{1}, y_{2}\right) \geq t$ for any $y_{1}, y_{2} \in Y$ with $y_{1} \neq y_{2}$. A $t$-approximation of $X$ is a maximal (with respect to the inclusion) $t$-separated set in $X$. 
Let $X$ be a complete Riemannian manifold with induced metric $d$. If $A_{\varepsilon}$ is an $\varepsilon$ approximation of $X$, the graph $\Gamma_{A_{\varepsilon}}=\left(A_{\varepsilon}, E\right)$ with $E:=\left\{x y: x, y \in A_{\varepsilon}, 0<d(x, y) \leq\right.$ $2 \varepsilon\}$ is called an $\varepsilon$-net of $X$.

Proposition 1 [37, Lemmas 2.5 and 4.5] If $X$ is a complete Riemannian manifold with bounded local geometry and $\Gamma$ is an $\varepsilon$-net in $X$, then $\Gamma$ and $X$ are quasi-isometric, and $h(X)>0$ if and only if $h(\Gamma)>0$.

Theorem 3 [32, p.88] If $X$ and $Y$ are quasi-isometric geodesic metric spaces, then $X$ is hyperbolic if and only if $Y$ is hyperbolic.

Proposition 2 [41, Proposition 5.6] If $X$ and $Y$ are quasi-isometric hyperbolic proper geodesic metric spaces, then $X$ has a pole if and only if $Y$ has a pole.

Lemma 1 [37, Lemma 2.3] Any E-net in a complete Riemannian manifold with bounded local geometry is uniform.

Theorem 4 [41, Theorem 5.12] If $X$ is a hyperbolic non-compact complete Riemannian manifold with bounded local geometry and a pole, then $h(X)>0$ if and only if $\partial_{\infty} X$ is uniformly perfect.

In [21] appear several sufficient conditions that guarantee that a Riemannian $n$-manifold is hyperbolic. Besides, see [53] for the case $n=2$ of Riemannian surfaces.

\section{Main results}

In this section we prove that having a pole is a necessary condition for a hyperbolic uniform graph or a complete Riemannian manifold with bounded local geometry satisfying isoperimetric inequality. Therefore, it can be removed as hypothesis in the statements of Theorems 2 and 4.

In fact, we are going to prove the following results.

Theorem 5 Given a hyperbolic uniform graph $\Gamma$, then $h(\Gamma)>0$ if and only if $\Gamma$ is an infinite graph with a pole and $\partial_{\infty} \Gamma$ is uniformly perfect for some visual metric.

Theorem 6 Let $X$ be a hyperbolic complete Riemannian manifold with bounded local geometry. Then, $h(X)>0$ if and only if $X$ is non-compact and has a pole and $\partial_{\infty} X$ is uniformly perfect.

These results are a consequence of Theorems 2, 4, and the two following results.

Theorem 7 Let $\Gamma$ be a uniform graph. If $\Gamma$ is hyperbolic and $h(\Gamma)>0$, then $\Gamma$ is an infinite graph with a pole.

Theorem 8 Let $X$ be a complete Riemannian manifold with bounded local geometry. If $X$ is hyperbolic and $h(X)>0$, then $X$ is non-compact and has a pole.

Let us now proceed with the proof of Theorem 7. 
Proof Seeking for a contradiction assume that $\Gamma$ is a finite graph. Since $h(\Gamma)>0$, if we consider the set $U=V(\Gamma)$, then

$$
0<h(\Gamma) \leq \frac{|\partial V(\Gamma)|}{|V(\Gamma)|}=0
$$

a contradiction. Therefore, $\Gamma$ is an infinite graph.

Seeking for a contradiction assume that $\Gamma$ does not have a pole. Let $\mu$ be a constant such that $\Gamma$ is $\mu$-uniform.

Fix $v \in V(\Gamma)$ and denote by $K$ the union of the geodesic rays starting from $v$. Note that there exists at least a geodesic ray, since $\Gamma$ is an infinite graph, and so $K \neq \emptyset$. Since $\Gamma$ does not have a pole, for each $n$ there exists $v_{n} \in V(\Gamma)$ with $d_{\Gamma}\left(v_{n}, K\right) \geq 4 n$. Let $\eta_{n}:\left[0, \ell_{n}\right] \rightarrow \Gamma$ be a geodesic joining $v_{n}$ with $K$ and such that $\eta_{n}(0)=v_{n}$ and $d_{\Gamma}\left(v_{n}, K\right)=L\left(\eta_{n}\right)=\ell_{n} \geq 4 n$.

Given $s \in \mathbb{R}$, denote by $\lfloor s\rfloor$ the lower integer part of $s$, i.e., the largest integer not greater than $s$. Assume that the ball $B_{\Gamma}\left(\eta_{n}(t),\left\lfloor\delta_{t h}(\Gamma)\right\rfloor+1\right)$ intersects every geodesic joining $v_{n}$ with some point of $K$, for some $t \in \mathbb{Z}^{+}$with $\left\lfloor\delta_{t h}(\Gamma)\right\rfloor<t \leq 4 n \leq \ell_{n}$. Thus, the connected component $A_{n}$ of $\Gamma \backslash B_{\Gamma}\left(\eta_{n}(t),\left\lfloor\delta_{t h}(\Gamma)\right\rfloor+1\right)$ containing $v_{n}$ satisfies that its boundary $\partial A_{n}$ is contained in the sphere $S_{\Gamma}\left(\eta_{n}(t),\left\lfloor\delta_{t h}(\Gamma)\right\rfloor\right)$ since $\Gamma$ is a graph.

Since $\Gamma$ is $\mu$-uniform,

$$
\left|\partial A_{n}\right| \leq\left|S_{\Gamma}\left(\eta_{n}(t),\left\lfloor\delta_{t h}(\Gamma)\right\rfloor\right)\right| \leq \mu^{\left\lfloor\delta_{t h}(\Gamma)\right\rfloor} .
$$

Thus

$$
\begin{aligned}
t & -\left\lfloor\delta_{t h}(\Gamma)\right\rfloor=\left|V(\Gamma) \cap \eta_{n}\left(\left[0, t-\left\lfloor\delta_{t h}(\Gamma)\right\rfloor-1\right]\right)\right| \\
& \leq\left|A_{n}\right| \leq h(\Gamma)^{-1}\left|\partial A_{n}\right| \leq h(\Gamma)^{-1} \mu^{\left\lfloor\delta_{t h}(\Gamma)\right\rfloor},
\end{aligned}
$$

and we conclude that

$$
t \leq\left\lfloor\delta_{t h}(\Gamma)\right\rfloor+h(\Gamma)^{-1} \mu^{\left\lfloor\delta_{t h}(\Gamma)\right\rfloor}=: M .
$$

Hence, if $2 n>M$, there exists a geodesic $\sigma_{n}$ from $v_{n}$ to $K$ such that $\sigma_{n} \cap$ $B_{\Gamma}\left(\eta_{n}(2 n),\left\lfloor\delta_{t h}(\Gamma)\right\rfloor+1\right)=\emptyset$. Note that $d_{\Gamma}\left(\eta_{n}(2 n), K\right)=\ell_{n}-2 n \geq 2 n$. Let $x_{n}$ and $y_{n}$ be the endpoints of $\eta_{n}$ and $\sigma_{n}$ in $K$, respectively, and consider the geodesic triangle $T^{n}=\left\{v_{n}, x_{n}, y_{n}\right\}$ in $\Gamma$. Since $\delta_{t h}(\Gamma)<\left\lfloor\delta_{t h}(\Gamma)\right\rfloor+1$, we have

$$
d_{\Gamma}\left(\eta_{n}(2 n), \sigma_{n}\right) \geq\left\lfloor\delta_{t h}(\Gamma)\right\rfloor+1>\delta_{t h}(\Gamma),
$$

and so, there exists $z_{n} \in\left[x_{n} y_{n}\right]$ with $d_{\Gamma}\left(\eta_{n}(2 n), z_{n}\right) \leq \delta_{t h}(\Gamma)$ (see Fig. 1).

Consider now the geodesic triangle $T_{n}=\left\{v, x_{n}, y_{n}\right\}$ in $\Gamma$, and $z_{n} \in\left[x_{n} y_{n}\right]$. Since $\left[v x_{n}\right] \cup$ $\left[v y_{n}\right] \subset K$, we have

$$
\begin{aligned}
& \delta_{t h}(\Gamma) \geq d_{\Gamma}\left(z_{n},\left[v x_{n}\right] \cup\left[v y_{n}\right]\right) \geq d_{\Gamma}\left(z_{n}, K\right) \\
& \quad \geq d_{\Gamma}\left(\eta_{n}(2 n), K\right)-d_{\Gamma}\left(\eta_{n}(2 n), z_{n}\right) \geq 2 n-\delta_{t h}(\Gamma),
\end{aligned}
$$

and so, $\delta_{t h}(\Gamma) \geq n$ for every $n$. This is the contradiction we were looking for, since $\Gamma$ is hyperbolic, and we conclude that $\Gamma$ has a pole.

Finally, let us now proceed with the proof of Theorem 8.

Proof Seeking for a contradiction assume that $X$ is a compact manifold. Since $h(X)>0$, if we consider the set $U=X$, then

$$
0<h(X) \leq \frac{\operatorname{Vol}_{n-1}(\partial X)}{\operatorname{Vol}_{n}(X)}=0,
$$




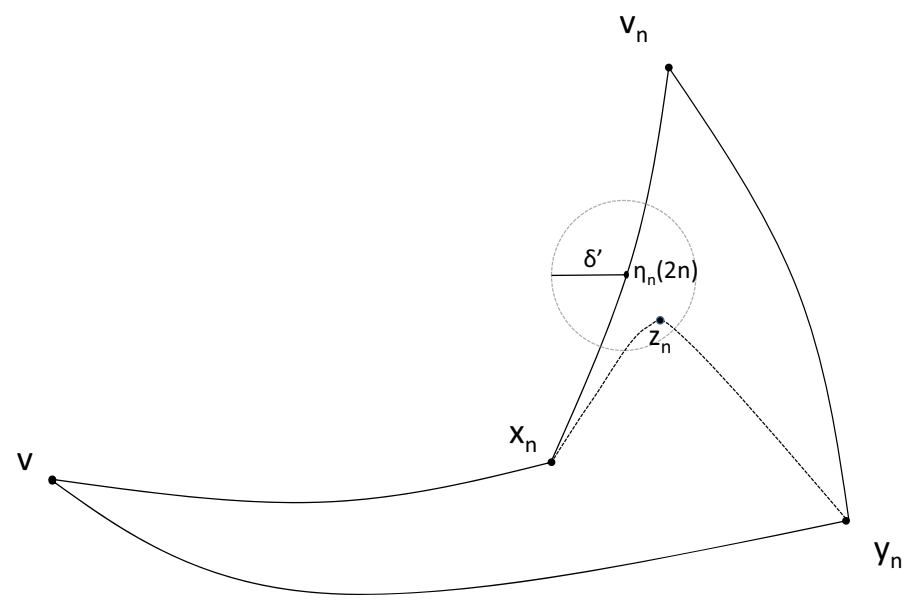

Fig. 1 If there is a geodesic $\sigma_{n}=\left[v_{n} y_{n}\right]$ from $v_{n}$ to $K$ which does not intersect the ball $B_{\Gamma}\left(\eta_{n}(2 n), \delta^{\prime}\right)$, where $\delta^{\prime}:=\left\lfloor\delta_{t h}(\Gamma)\right\rfloor+1$, then there is a point $z_{n} \in\left[x_{n} y_{n}\right]$ which is far from $K$

a contradiction. Hence, $X$ is non-compact.

Let $\Gamma$ be an $\varepsilon$-net in $X$. Since $h(X)>0$, Proposition 1 gives that $h(\Gamma)>0$.

Note that $X$ is a proper geodesic metric space since it is a complete Riemannian manifold. Also, $\Gamma$ is a proper geodesic metric space since it is a uniform graph by Lemma 1 .

By Proposition 1, $X$ and $\Gamma$ are quasi-isometric and so, $\Gamma$ is hyperbolic by Theorem 3 . Theorem 7 gives that $\Gamma$ has a pole, and by Proposition 2, $X$ has a pole.

Remark 1 Theorem 5 also allows to improve Theorem 4.12 in [42] which is based in the same results. In the same way, having a pole is a necessary condition and can be removed as hypothesis.

Remark 2 Theorem 5.11 in [44] states that for geodesic, visual, Gromov hyperbolic spaces, having uniformly perfect boundary at infinity is equivalent to being uniformly equilateral. Thus, Theorems 5 and 6 can also be written using this property and without using the boundary at infinity.

Acknowledgements We would like to thank to the referees for their careful reading and helpful comments.

Funding Open Access funding provided thanks to the CRUE-CSIC agreement with Springer Nature.

Open Access This article is licensed under a Creative Commons Attribution 4.0 International License, which permits use, sharing, adaptation, distribution and reproduction in any medium or format, as long as you give appropriate credit to the original author(s) and the source, provide a link to the Creative Commons licence, and indicate if changes were made. The images or other third party material in this article are included in the article's Creative Commons licence, unless indicated otherwise in a credit line to the material. If material is not included in the article's Creative Commons licence and your intended use is not permitted by statutory regulation or exceeds the permitted use, you will need to obtain permission directly from the copyright holder. To view a copy of this licence, visit http://creativecommons.org/licenses/by/4.0/.

\section{References}

1. Abu-Ata, M., Dragan, F.F.: Metric tree-like structures in real-life networks: an empirical study. Networks 67, 49-68 (2016) 
2. Adcock, A.B., Sullivan, B.D., Mahoney, M.W.: Tree-like structure in large social and information networks. In: 13th International Conference Data Mining (ICDM), pp. 1-10. IEEE, Dallas, Texas, USA (2013)

3. Alonso, J., Brady, T., Cooper, D., Delzant, T., Ferlini, V., Lustig, M., Mihalik, M., Shapiro, M., Short, H.: Notes on word hyperbolic groups. In: Ghys, E., Haefliger, A., Verjovsky, A. (eds.) Group Theory from a Geometrical Viewpoint. World Scientific, Singapore (1992)

4. Alvarez, V., Pestana, D., Rodríguez, J.M.: Isoperimetric inequalities in Riemann surfaces of infinite type. Rev. Mat. Iberoam. 15, 353-427 (1999)

5. Alvarez, V., Portilla, A., Rodríguez, J.M., Tourís, E.: Gromov hyperbolicity of Denjoy domains. Geom. Dedicata 121, 221-245 (2006)

6. Alvarez, V., Rodríguez, J.M., Yakubovich, D.V.: Estimates for nonlinear harmonic "measures" on trees. Mich. Math. J. 49, 47-64 (2001)

7. Ancona, A.: Negatively curved manifolds, elliptic operators, and Martin boundary. Ann. Math. 125, 495-536 (1987)

8. Ancona, A.: Positive harmonic functions and hyperbolicity. In: Král, J., et al. (eds.) Potential Theory, Surveys and Problems. Lecture Notes in Math, vol. 1344, pp. 1-24. Springer, Heidelberg (1988)

9. Ancona, A.: Theorie du potentiel sur les graphes et les varieties. In: Ancona, A., et al. (eds.) Ecolé d'Eté de Probabilités de Saint-Flour XVII-1988. Lecture Notes in Math, vol. 1427. Springer, Heidelberg (1990)

10. Anderson, M., Cheeger, J.: $C^{\alpha}$-compactness for manifolds with Ricci curvature and injectivity radius bounded below. J. Differ. Geom. 35, 265-281 (1992)

11. Bermudo, S., Rodríguez, J.M., Sigarreta, J.M.: Computing the hyperbolicity constant. Comput. Math. Appl. 62, 4592-4595 (2011)

12. Bermudo, S., Rodríguez, J.M., Sigarreta, J.M., Tourís, E.: Hyperbolicity and complement of graphs. Appl. Math. Lett. 24, 1882-1887 (2011)

13. Bishop, C.J., Jones, P.W.: Hausdorff dimension and Kleinian groups. Acta Math. 179, 1-39 (1997)

14. Bridson, M., Haefliger, A.: Metric Spaces of Non-positive Curvature. Springer, Berlin (1999)

15. Brinkmann, G., Koolen, J., Moulton, V.: On the hyperbolicity of chordal graphs. Ann. Comb. 5, 61-69 (2001)

16. Burago, D., Burago, Y., Ivanov, S.: A Course in Metric Geometry. Graduate Studies in Mathematics, vol. 33. AMS, Providence (2001)

17. Buser, P.: A note on the isoperimetric constant. Ann. Sci. École Normale Sup. 15, 213-230 (1982)

18. Buser, P.: Geometry and Spectra of Compact Riemann Surfaces. Birkhäuser, Boston (1992)

19. Buyalo, S., Schroeder, V.: Elements of Asymptotic Geometry. EMS Monographs in Mathematics, Berlin (2007)

20. Cantón, A., Granados, A., Portilla, A., Rodríguez, J.M.: Quasi-isometries and isoperimetric inequalities in planar domains. J. Math. Soc. Jpn. 67, 127-157 (2015)

21. Cao, J.: Cheeger isoperimetric constants of Gromov-hyperbolic spaces with quasi-pole. Commun. Contemp. Math. 4(2), 511-533 (2000)

22. Chavel, I.: Isoperimetric Inequalities: Differential Geometric and Analytic Perspectives. Cambridge University Press, Cambridge (2001)

23. Cheeger, J.: A lower bound for the smallest eigenvalue of the laplacian. In: Gunning, R.C. (ed.) Problems in Analysis, pp. 195-200. Princeton University Press, Princeton (2015). https://doi.org/10.1515/ 9781400869312-013

24. Clauset, A., Moore, C., Newman, M.E.J.: Hierarchical structure and the prediction of missing links in networks. Nature 453, 98-101 (2008)

25. Dress, A., Holland, B., Huber, K.T., Koolen, J.H., Moulton, V., Weyer-Menkhoff, J.: $\Delta$ additive and $\Delta$ ultra-additive maps, Gromov's trees, and the Farris transform. Discrete Appl. Math. 146(1), 51-73 (2005)

26. Dress, A., Moulton, V., Terhalle, W.: T-theory: an overview. Eur. J. Comb. 17, 161-175 (1996)

27. Fernández, J.L., Melián, M.V.: Bounded geodesics of Riemann surfaces and hyperbolic manifolds. Trans. Am. Math. Soc. 347, 3533-3549 (1995)

28. Fernández, J.L., Melián, M.V.: Escaping geodesics of Riemannian surfaces. Acta Math. 187, 213-236 (2001)

29. Fernández, J.L., Melián, M.V., Pestana, D.: Quantitative mixing results and inner functions. Math. Ann. 337, 233-251 (2007)

30. Fernández, J.L., Melián, M.V., Pestana, D.: Expanding maps, shrinking targets and hitting times. Nonlinearity 25, 2443-2471 (2012)

31. Fernández, J.L., Rodríguez, J.M.: The exponent of convergence of Riemann surfaces: bass Riemann surfaces. Ann. Acad. Sci. Fenn. A I(15), 165-183 (1990)

32. Ghys, E., de la Harpe, P.: Sur les Groupes Hyperboliques d'après Mikhael Gromov. Progress in Mathematics, vol. 83. Birkhäuser, Berlin (1990) 
33. Granados, A., Pestana, D., Portilla, A., Rodríguez, J.M., Tourís, E.: Stability of the injectivity radius under quasi-isometries and applications to isoperimetric inequalities. RACSAM 112, 1225-1247 (2018)

34. Gromov, M.: Hyperbolic groups. In: Gersten, S.M. (ed.) Essays in Group Theory, M. S. R. I. Publ, vol. 8, pp. 75-263. Springer, Heidelberg (1987)

35. Jonckheere, E.A.: Contrôle du traffic sur les réseaux à géométrie hyperbolique-Vers une théorie géométrique de la sécurité l'acheminement de l'information. J. Eur. Syst. Autom. 8, 45-60 (2002)

36. Jonckheere, E.A., Lohsoonthorn, P.: Geometry of network security. American Control Conference ACC, pp. 111-151 (2004)

37. Kanai, M.: Rough isometries and combinatorial approximations of geometries of noncompact Riemannian manifolds. J. Math. Soc. Jpn. 37, 391-413 (1985)

38. Krioukov, D., Papadopoulos, F., Kitsak, M., Vahdat, A., Boguña, M.: Hyperbolic geometry of complex networks. Phys. Rev. E 82(3), 036106 (2010)

39. Martínez-Pérez, A.: Chordality properties and hyperbolicity on graphs. Electron. J. Comb. 23(3), P3.51 (2016)

40. Martínez-Pérez, A.: Generalized chordality, vertex separators and hyperbolicity on graphs. Symmetry 9(10), 199 (2017)

41. Martínez-Pérez, A., Rodríguez, J.M.: Cheeger isoperimetric constant of Gromov hyperbolic manifolds and graphs. Commun. Contemp. Math. 20(5), 1750050 (2018)

42. Martínez-Pérez, A., Rodríguez, J.M.: Isoperimetric inequalities in Riemann surfaces and graphs. J. Geom. Anal. 31, 3583-3607 (2021)

43. Melián, M.V., Rodríguez, J.M., Tourís, E.: Escaping geodesics in Riemannian surfaces with pinched variable negative curvature. Adv. Math. 345, 928-971 (2019)

44. Meyer, J.: Uniformly perfect boundaries of Gromov hyperbolic spaces. Ph.D. thesis, University of Zurich (2009)

45. Montgolfier, F., Soto, M., Viennot, L.: Treewidth and hyperbolicity of the internet. In: 10th IEEE International Symposium on Network Computing and Applications (NCA), pp. 25-32 (2011)

46. Paulin, F.: On the critical exponent of a discrete group of hyperbolic isometries. Differ. Geom. Appl. 7, 231-236 (1997)

47. Pestana, D., Rodríguez, J.M., Sigarreta, J.M., Villeta, M.: Gromov hyperbolic cubic graphs. Cent. Eur. J. Math. 10, 1141-1151 (2012)

48. Pólya, G.: Isoperimetric Inequalities in Mathematical Physics. Princeton University Press, Princeton (1951)

49. Shang, Y.: Lack of Gromov-hyperbolicity in colored random networks. Pan Am. Math. J. 21(1), 27-36 (2011)

50. Shang, Y.: Lack of Gromov-hyperbolicity in small-world networks. Cent. Eur. J. Math. 10(3), 1152-1158 (2012)

51. Shang, Y.: Non-hyperbolicity of random graphs with given expected degrees. Stoch. Models 29(4), 451462 (2013)

52. Sullivan, D.: Related aspects of positivity in Riemannian geometry. J. Differ. Geom. 25, 327-351 (1987)

53. Tourís, E.: Graphs and Gromov hyperbolicity of non-constant negatively curved surfaces. J. Math. Anal. Appl. 380, 865-881 (2011)

54. Väisälä, J.: Hyperbolic and uniform domains in Banach spaces. Expos. Math. 23(3), 187-231 (2005)

55. Wu, Y., Zhang, C.: Chordality and hyperbolicity of a graph. Electr. J. Comb. 18, P43 (2011)

Publisher's Note Springer Nature remains neutral with regard to jurisdictional claims in published maps and institutional affiliations. 